\title{
Peer Learning Methodology for Sustainable Energy Usage
}

\author{
Frans Folkvord ${ }^{1,2(\bowtie)}$, Lutz Peschke ${ }^{3}$, Gökçen Baș ${ }^{4}$, Simone Vitiello ${ }^{1}$, \\ and Nathan Spunda ${ }^{1,2,3,4}$ \\ ${ }^{1}$ Open Evidence Research, Barcelona, Spain \\ \{ffolkvord, svitiello\}@open-evidence.com, \\ nathan.spunda@gmail.com \\ 2 Tilburg School of Humanities and Digital Sciences, Tilburg, The Netherlands \\ ${ }^{3}$ Department of Communication and Design, Bilkent University Ankara, \\ Ankara, Turkey \\ lutz.peschke@bilkent.edu.tr \\ ${ }^{4}$ Vienna University of Technology, Vienna, Austria \\ goekcen.bas@tuwien.ac.at
}

\begin{abstract}
Humanity must be aware of the possibilities for sustainable energy technologies from their childhood onwards in order to enable a clean and prosperous future. Until now, youth receives only limited and mostly theoretical knowledge about new forms of energy usage, which leaves open doors to the usage of conventional energy sources. It is vital that youth develops a solid understanding of renewable energy's power and its various possible applications, thereby taking into account. Currently, youth are getting increasingly used to learn through transactional forms of communication via their (online) media consumption behaviour and contact with peers through social media. Considering these new communication forms, this study examines if peer-learning methodology (PLM) is an efficient method to train children in increasing their practical knowledge of sustainable energy usage. Nowadays, PLM is used as an educational methodology based on an eclectic integration of multiple theoretical insights from different scientific disciplines, such as developmental psychology, education science and paediatrics, that might be an effective learning methodology. The main idea behind PLM is that in order to educate youth effectively, a communication must motivate the receiver to actively attend to messages and perceive and interpret their content that is provided by peers, include iterative and transactional solicitation of feedback, and activate elaboration of message arguments and counterarguments to encourage individuals to move through the process of learning. In the current study, we investigated how students learn from other peers that have made posters that reflect on new and sustainable forms of energy. In total 14 posters and four movies were shown during an exhibition in both Ankara (Turkey) and in Tilburg (the Netherlands). During the exhibition, 30 pairs of students in Ankara and 12 pairs of students in Tilburg were equipped with a GoPro and they should talk about the exhibition according to the think aloud method. This data will be recoded afterwards by trained researchers in order to establish participants responses.
\end{abstract}

Keywords: Sustainable energy $\cdot$ Peer-learning methodology $\cdot$ Education · New forms of communication 


\section{Introduction}

Access to clean, affordable and reliable energy has been a cornerstone of the world's increasing prosperity and economic growth since the beginning of the industrial revolution [1]. For some decades, human activity is overloading our atmosphere with carbon dioxide and other global warming emissions, which trap heat, steadily drive up the planet's temperature, and create significant and harmful impacts on global health, environment, and climate $[2,3]$. Therefore, the use of energy by the global population in the twenty-first century and future generations must become sustainable to be able to fulfil upcoming generations' energy-needs, in particular taking into account the increasing energy-need for developing countries [4]. The world needs another energy revolution in which the sources of energy are affordable, accessible, and sustainable $[2,3]$. Energy efficiency and conservation, as well as decarbonizing the energy sources, are essential elements in this revolution.

Different initiatives have been launched and agreements have been set in order to increase the usage of sustainable energy. The most apparent and recent initiative is the Paris agreement, that has been ratified globally by practically every country, except the US and North Korea [5]. The agreement, states that society- and economy-wide decisions should be conducted to absolute emission reduction targets. Exposure to air pollutants as a consequence of traditional energy usage, like nitrogen oxides have been identified as a significant risk factor for a number of health conditions including extinction of species, respiratory infections of humans, heart disease, stroke and lung cancer [5,6]. At the moment, greenhouse gas emissions still remain above agreed levels and would need to fall dramatically in order to meet international agreements [5]. In order to comply with the directives agreed on in the Paris Agreements, participating countries will possibly be obliged to take green initiatives to change behaviour and reduce air pollution in large cities and highly trafficked areas [7].

Solar, wind and water-based energy systems, and engineering of microbes to produce biofuels, are only a few examples that have been developed recently and can replace traditional forms of energy usage, in order to reduce the emissions and have positive benefits on world's energy security and the environment [8]. In addition, an increasing number of studies convincingly shows that renewable energy consumption benefits not only the environment but also for the economic conditions of (developing) countries [4, 9, 10]. Although an increasing number of sustainable energy-forms become apparent, it will take ambitious efforts to increase the actual implementation of these new techniques in everyday life.

Humanity must be aware of their existence, potentials, harvesting, technologies of use and benefits from very childhood onwards in order to enable clean and prosperous future. Until now, youth receives only limited theoretical knowledge about new forms of energy usage, which leaves open doors to continue consuming conventional energy sources. Renewable energies are available everywhere and most of the technologies of application are mature and relatively simple to implement in everyday life. It is vital that youth develops a solid understanding of renewable energy's power and its various possible applications, thereby taking into account effective educational strategies to educate children about sustainable energy usage, both on a theoretical and practical level. 
Nowadays, youth are getting increasingly used to more transactional forms of communication through their online behaviour than the more traditional forms of communication, that are considered as one-directional [11]. To adapt the educational system in parallel to this new form of communication, the peer-learning methodology (PLM) has recently been established. In addition to this, there is a need to attract youth for the theme of environmental protection and conservation of nature through the use of renewable energy technologies. This study will address if peer-learning methodology is an effective method to publicly share information and knowledge about renewable energy technologies in an entertaining way. The current study tests if PLM can be used as a great start for youngsters to learn more about all types of renewable energy, in order to grow up more informed about the vital role of renewable technologies in changing world's energy future. Before we explain the methodology of the study and show the results, we will first elaborate more on PLM and about the theoretical underpinnings underlying PLM.

\subsection{Peer Learning Methodology (PLM)}

Every year new challenges emerge in the field of training and development, like competency development, outsourcing, e-learning, and knowledge management. The common theme of all these educational developments is that businesses, teachers, and political leaders want to assess and see value for their investment in educational programs, not only in improving the theoretical understanding of certain topics by children, but also on the practical understanding of children about what should be done. Core outcomes for educational programs that need to be assessed are if the learning is relevant and immediately applicable to their needs, and if the learning is effective and sustainable.

Different studies have shown that PLM is an effective tool to guarantee a high rate for the use of quality resources and the accuracy of content [12]. Furthermore, peer assessments encouraged feedback to the trainee leading to a learning arena of the collaborative learning. In the current study we test the use of the PLM, which is an innovative learning methodology financed by the European Commission [12]. The main aspect of PLM is that students learn from others (peers) by actively engaging in large trainee groups to experience practical skills, in combination with appropriate and real time feedback in order to improve their deep understanding and learning of realworld aspects. PLM is one the most efficient method among children and young people, therefore our aim is, under this project, to test PLM as an efficient method to reach a bigger number of children and young people and, therefore, to create a higher level of awareness on renewable energy.

\subsection{Theoretical Underpinnings}

The PLM is based on an selective integration of multiple theories from (social and developmental) psychology, paediatrics and education science. For example, the SelfDetermination Theory [13] states that people have three innate needs; (1) competence, (2) feeling of relatedness and (3) autonomy. In addition, the Self-Determination Theory argues that (1) people are proactive with their potential and mastering their inner forces 
(such as drives and emotions), (2) people have an inherent tendency toward growth development and integrated functioning, and (3) people strive for optimal development and actions, that are inherent in people but they don't happen automatically and should be supported/triggered by the (social) environment. The self-determination theory focuses in particular on the degree to which an individual is self-motivated and selfdetermined or influenced by other factors. In addition, the Social Cognitive Theory [14] states that seeing or visualizing people (modelling) similar to oneself, like peers, perform successfully typically raises beliefs in observers that they themselves possess the capabilities to master comparable activities. This is called observational/vicarious learning, thereby affecting (1) self-efficacy, (2) outcome expectancies, (3) behaviour capabilities, (4) self-control, (5) managing emotional arousal, and (6) reciprocal determinism. In sum, the main idea behind PLM is that in order to educate youth effectively, a communication must motivate the receiver to actively attend to messages and perceive and interpret their content that is provided by peers, include iterative and transactional solicitation of feedback, and activate elaboration of message arguments and counterarguments to encourage individuals to move through the process of learning.

The current study uses PLM to increase youngster competence in renewable energy through innovative and creative learning. Furthermore, it aims to train young people to be able to organize their community for launching a local renewable energy initiative with bottom up approach and to train young people's to be able to select the technologically, agriculturally, economically, socially and environmentally best fitting option for their community. As a consequence, it will increase young peoples' competence in renewable energy through innovative and creative learning, and be able to train the local actors to be able to organize their community for launching a local renewable energy initiative with bottom-up approach - train the local actors to be able to select the technologically, agriculturally, economically, socially and environmentally best fitting option for their community. The general aim of the study is to raise awareness about the importance of adopting sustainable habits and behaviours. We aim at making young people and the audience feel more concerned about these issues and make them more eco-responsible citizens. Hereby we hope that the young people realize that some problems concerning sustainability can be dealt with on a local and even personal level. Hence developing a sense of how they can convince others to act in a sustainable way. Partial aims of the study are (1) acquiring practical skills, (2) Obtaining professional skills, (3) accepting environmental values, (4) improving language skills, (5) social and cultural competences.

\section{Reception Study in Ankara (Turkey) and Tilburg (the Netherlands) About an Environmental Science Exhibition}

Aiming to understand peer learning strategies of young people in the context of environmental science communication, an exhibition about renewable energy and related environmental issues were created and a reception study was executed in Ankara and Tilburg. The study was verified in two parts. 


\subsection{Creating Popular Science Media}

In the first part, exhibits were created within the undergraduate course "Science Writing and Journalism" at Bilkent University, Ankara. Fifteen students prepared text and graphic posters based on data which were provided by Vienna University of Technology and the Provincial Directorate of Environment and Urbanization in Ankara. Additionally, the students searched for other relevant scientific sources. The students were instructed to create media with a high content of storytelling which supports the recipients to understand complex scientific topics in a more sustainable way. The posters could be divided into three different categories. One set of posters dealt concretely with specific topics of energy generation: renewable energies, like geothermal power, wind energy, hydroelectric power as well as nuclear power. Another group of posters presented aspects of power, like how is the renewable energy concept in Arabian countries, used and not used potentials of renewable energy in Turkey, or does Turkey need more energy plants. Other posters discussed topics which are related with climate change and environmental issues, like the relation of urbanization and rainfall, plastic bags and meat consumption as well as the mind experiments about usage of renewable energy on Mars as well as about the question, would Neanderthals live more in harmony with the nature, if they would live today. All posters were accompanied by popular scientific texts.

The posters with specific renewable energies were added with schemes about technical aspects of power plants, which were produced by the project partner iserundschmidt from Bonn/Germany. The methodology of the poster and text creation is based on the approach that media which are received during leisure time will awaken more general and intrinsic interest, but also generate voluntary motivated relevance of youth. Peschke [15] developed a reception model which considers that knowledge can be received in a sustainable way when the topic is connected with own experiences of the life world. It includes Luhmann's theory of social systems [16] and the aspect of Schütz and Luckmann's life world [17].

Schütz's approach connects the everyday life world and the scientific world of knowledge. On the other hand, Habermas [18] understands as the structural components of the life world culture, society and person. The correlation of Habermas' models of the life world with Luhmann's system theory, where science is a sub-system of society, leads to the model where science is a part of the life world. But science is outside of the stock of knowledge which participants of communication use to agree on an object of the world. According to Habermas, this stock of knowledge is located in the culture. As a consequence, media has to contribute to the popularization of science, which is a process of decreasing the distance between science world and life world in order to share knowledge and influence the mass [17] and is the mediatized process of enabling the communicative interaction between science and culture.

The premise of this reception model [15] is, that science as a system has no access to the communicative everyday life practice without medial support. The function of media is, to enable catalytically a compatibility between the scientific object and existing mental images. Thereby, a translation process occurs, which initiates a compatible operation in the system culture - according to Habermas part of the life world. The personal relevance place an important role in this context. In this context, 
relevance is understood as pertinence in accordance with Foskett [19] and Lancaster [20], regarding the relationship between a medium and an information need. According to the authors, the global information need is higher than the individual information need, because of the big distance between lifeworld and the science world. Since there is no authority which can force an individuum to acquire knowledge, the existence of a voluntarily motivated relevance to deal with a certain scientific topic is needed.

For the reception study arise the following consequence: According to Foskett and Lancaster, the relevance is the relation between information need and medium. If there is an information need according to a topic, the relevance will be evaluated and verified with the help of voluntarily reception of a medium. If there is no information need, there cannot occur a verification of relevance. But a medium is able to generate interest to deal with it. But interest is not sufficient enough to start a process of reception. Otherwise, a medium would have to be only attractive to catch the recipient and the reception would be proceeded automatically, which is not the case. Accordingly, beside the interests, there has to exist an information need to deal with a scientific topic, which means that, a medium is only regarded as relevant with respect of the acquisition of knowledge, if a recipient has an information need which has to be released through awakening interest, dependent on the distance to the everyday life. But this approach is only viable if we assume in accordance with Mizzaro [21] that an information need is not a static and unchanging need but will change over the reception process dependent on the media. Mizzaro calls it a perceived information need. As a consequence, media have potentially impact on the individual perceived information need and can affect relevance, which is in harmony with Hall's representation approach [22].

Considering the approach described above, the students of the Department of Communication and Design at Bilkent University created twelve graphic posters with diagrams, pictures and verbal texts as well as popular scientific text posters [23]. The major types of renewable energy sources that were used were defined as solar, wind, geothermal, hydropower, ocean, tidal, biomass are studied over the last decades for a broad view of feasibility, development and integration in each category and were therefore used in the current study [24]. Students used the relevant literature comprising not only technical knowledge but also environmental relationship, economic and social effects of sustainable energy use. The exhibition additionally consisted of schemes about renewable energies as well as two animation movies about embodied energy and hydropower, which were produced by iserundschmidt - agency for science communication in Bonn, Germany.

\section{Methodology}

The reception study was conducted with 60 students in Ankara and 24 students in Tilburg. The participants consist of 34 young man and 48 young women. The average age of them was 21,15 years $(\mathrm{N}=84$; $\mathrm{SD}=2,5$; Median $=21)$. The participants were all students that were doing the following studies: Information Technologies $(\mathrm{N}=12)$, Engineering $(\mathrm{N}=6)$, Science $(\mathrm{N}=5)$, Management $(\mathrm{N}=18)$ Social Sciences $(\mathrm{N}=11)$, Fine Arts, Design and Architecture $(\mathrm{N}=24)$ and other subjects $(\mathrm{N}=8)$. Pairs of students were asked to walk through the exhibition and to find out the main 
information of the different media. The study was conducted in a hallway at the campus of both universities. Participants were equipped with GoPro cameras which opened the opportunity to see how they moved through the exhibition, which details they checked, and the context of communication while decoding the information. Besides the two GoPro cameras, a wide-angle camera was placed which showed additionally the way which the pairs of participants chose in the exhibition. The pairs of participants were informed that they were completely free to decide in which order they analyse the exhibits, how long they wanted to deal with the different media as well as the way and context in which they wanted to discuss the provided topics. The only task which was given by the supervisor was that they should try to find out the main idea of the provided media, that they should talk about what they think, use the English language during the process of communication, and that they should stay always together.

The reception study is based on mainly two approaches. The task that the participants should talk as much as they can about what they think is related to the Think Aloud Method, developed and described by van Someren et al. [25]. The think aloud method is originally developed to observe participants while solving a given problem and to analyse the resulting verbal protocols. It is mainly applied in psychological and educational research on cognitive processes but also for knowledge acquisition (ibid,7). This method has its origin in the psychological research developed from the so-called introspection methods. Introspection is grounded in the idea "that one can observe events that take place in consciousness, more or less as one can observe events in the outside world." (ibid., 29). The advantage is that the produced data are open to analysis and interpretation for anyone. Within the scope of the reception study, the think aloud method is not used in the original way as described by van Someren et al. Instead of given a concrete problem which should be solved during the study, the participants were completely free to decide in which context they want to discuss the certain media. The aim was not to solve a concrete problem but to produce own constructs related with their individual constructed world. This strategy is closely related to Kelly's personal construct psychology. The Personal Construct Psychology is a fundamental work by Kelly [26] which is based on the constructivist approach that the world can only be identified by a human being with help of identification, evaluation and interpretation of certain events in the relation of his/her subjective world. It means that reality is not absolute truth but is based on alternative interpretations, which he/she can choose [27]. The evaluation can differ from human to human, and facts which are generated by these evaluations can be part of alternative constructs as well. Therefore, this philosophical position is described as constructivist alternativism [28]. The relation of the applied methods of the reception study to Kelly's personal construct psychology is generated by the strategy that no concrete question is asked to the participants, and that it is left open what the participants want to discuss. The freedom that the participants can choose the context how they want to discuss the given medium enables to get information about the constructs which are produced by the participants. It avoids that participants deal with constructs which are given by the supervisors. In the worst case the given constructs are not relevant with the life world of the participants. 


\section{Results}

With the help of four examples of the poster exhibitions in Ankara and Tilburg, the effect of PLM will be discussed. The first example is a graphic poster and a text from Seyfafjehi [29] about energy resources on Mars. The text was written in a screenplay style. Professor Neutron and Professor Sun have a fight about the best energy source for living on Mars. Both confront the other the negative sides and disadvantages of solar power, which is defended by Ms Sun, and nuclear power, which is defended by Mr Neutron. The dispute is finished by the interruption of Professor Steam who gives a statement in favour of geothermal energy. The graphic poster visualises the comparison of how solar, nuclear and geothermal power works on Earth and how it would work on Mars with the help of diagrams, schemes and verbal texts.

The participants 1A and 1B remained on that poster 14'19 min which was extraordinary long comparing with the other pairs of participants (almost between 1 and $5 \mathrm{~min}$ ). The following part of the dialogue shows a typical way of getting access to the information:

1B: So geothermal power is o.k., good. But, how can...

1A: It says here (points the scheme of geothermal energy on the lower left part of the graphic poster)

1B: How can it be renewable?

1A: Because water is off, always water, when you inject water, there, okay, because here in the core, it's after one, I mean.

1B: After a while, it will be down.

1A: Yes, it will be down, but here in the core, there is this energy, that doesn't go that, doesn't go away and when you start, when you inject...

1B: It does not finish...

1A: No, it does not finish. and when you send the water back, here, it heats again and again and again and you always use that steam, I mean, think about the core, it's really big and it's really hot especially on Earth, because, (points to the image on the upper left part of the graphic poster) cooler crusts, Mars has cooler crusts and core.

1B: Yeah, o.k., I see.

1A: And here you have, you don't have to drill so deep, because here is also hots, but on Mars, you have to deep drill, you, like, it stays in here to get hot water.

IB: I see. Actually, on Earth, in Turkey steam comes from the Earth.

1A: Yes, yes, and think about...

1B: Actually, my family lives in Meydan. And there are Geothermal energy stations. Yeah, to produce electric. And it is really close to surface.

The dialogue reveals significant information about the peer learning effect. In this part, participant 1B tries to understand the renewable aspect of geothermal energy. 1A got access to the topic obviously faster and explains it to 1B. It shows the two effects of peer learning: One part gets access to the information with the help of the explanation of the partner. The other partner gets access to the information by explaining step-bystep. Additionally, the two partners demonstrate the strategy of understanding through 
connecting the topic with stories and experiences from their own life world. Participant 1B obviously understood the topic that Mars has cooler crust and core and you would have to drill deeper to use geothermal power. At that moment, the participant compared this fact with the situation in her home town Meydan where geothermal power can be used in a quite efficient way. In a similar way, when they discussed the comments of Professor Neutron and the potential of nuclear power on Mars, participant 1A talked about a caesium analysis as a high school experiment, around 20 years after the nuclear disaster in Chernobyl.

"And I also made a project when I was in high school, I was in Trabzon, and there is a material, there is an element, Caesium, it only can be after a nuclear, nuclear, say, explosion explosion, and after Chernobyl, Caesium came to our land and when I dig the soil in Trabzon and examine it in the laboratory in university we found not high but low Caesium in the soil, so it still affects our soil and we're in Turkey".

The dialogue and quote indicate and confirm on the one hand the importance of storytelling for the Public Understanding of Science. Stories bridge scientific knowledge with the life world in a catalytic way. From the view of popularisation, it can be understood as the decrease in distance between knowledge from the science world and the experiences of the life world [23]. On the other hand, autonomous decisions of how to deal with the knowledge media reveal information about the constructed world of the participants, especially about their perceived information needs, interests and relatedness to the medium and information itself. Consequently, the peer learning method can be evaluated in the context of reception model based on the perceived information need. Finally, it is obvious that partners which discuss in a self-determined way learn scientific topics more sustainable.

The second example supports this hypothesis. It is a dialogue about a poster which communicates the principle of wind turbines. In the focus of the text and graphics poster is the harmful effect of the wind turbines for birds. Text and graphic poster inform about the fact that every year worldwide 140,000 until 328,000 birds die from the collision with wind turbines, especially which are located on their migration routes. At the same time, it communicates possible solutions to avoid the death of birds [30]. As followed, the dialogue of the participants no. 3 shows a more balanced way of peer learning. The pair remained 5,27 min at that set of posters which is nearly the average of the remaining time of the participants:

3B: I didn't know, that Turkey was in any migration route like, you see that?

3A: Yeah, but, but maybe, I don't think there are many ways within Turkey, like, routes of birds inside on, the inside Turkey, so it means that this is not a problem, they should consider, right?

3B: Yeah. (read)

3A: Yeah, exactly. Very important point.

3B: Yeah. but like you have to consider, that would, it affects wind farm's productivity, if they are located away from the migration routes, because migration routes would, the wind be higher on migration routes, or not?

3A: You're right, it helps them, actually you're right.

3B: Yeah.

3A: But it can't be like his that 
3B: I don't think, that is much of the problem, because 328,000 birds die from each..., ok. I don't think this number is not that huge, because it is across the world, I don't think, this is only in Turkey, is it?

3A: No, I don't think so, it's all over the world

3B: 328,000 birds dying is, like, probably one percent of the whole population of the birds dying,

3A: Well it can't be, I think the problem, they just focus on, is this problem

3B: Yeah.

3A: Of that temperature. That the birds are changing their roads and destination just because of their temperature was, which is getting higher

3B: Oh, they're like doing this in seas as well like

3A: Off-shore.

3B: In the oceans.

3A: I've never seen that, to be honest.

3B: I've never seen that since I've never been to the ocean. I've never seen ocean wind farms. that's interesting idea

3A: For ... there are some opinions, that we must really destroy the landscape, like, they destroyed the way, we perceive the landscape, like, the natural beauty the natural landscape, but I think it's not a really important factor.

Comparing with the dialogue of participants no.1, the dialogue of the participants no. 3 are more balanced in a way, that there is not one who explains the other the problems and facts which are provided of the poster. The interaction of the two partners is more shaped by a common discovery of the information. The peer learning strategy in the context of self-determination is more significant since the role of the teacher is dissolved. The learning strategy is negotiated between the partners in an emancipated way and less influenced by a concrete problem of one of the partners.

The two other posters of Akınc1 [31] and Cansaran [32] had two different kind of eyecatchers which enabled the entrance to the posters. Ak1nc1 created a poster about the comparison of rainfall between the small city Edirne inside of a rural area and the mega city Istanbul. Both cities are located in the same region of Turkey, called Marmara. The distance between both cities is around $240 \mathrm{~km}$. Without anthropogenic influences, the climate conditions should be very similar. But in one region of Istanbul the annual rainfall is extraordinary high caused by mirror-glass equipped skyscrapers and of the density of car traffic. Cansaran analysed the forecasts and real energy demands in Turkey. He reveals that the forecasts are much higher than the real needs. The design of infographics of both posters are very different. Cansaran's poster contains tables, text and pure bar charts. It follows the strategy and demand of Tufte in a consequent way. In contrast, Akınc1 presented her topics with embellished elements. Her poster is divided in three vertical sections. The upper section shows statistics about the numbers of cars in Edirne and Istanbul, as well as the number of skyscrapers and high buildings in Istanbul. The lower part visualises the population in Edirne and Istanbul. The diagrams and charts are embellished with symbols. They visualise the meaning of the diagram according to Neurath's strategy. Neurath used for his charts a mixture descriptive and analytical codes. The charts, like bar charts are in general very abstract representations and get a concrete meaning with addition of symbols. Neurath [33] called his mixed 
codes ISOTYPE (International System of Typographic Picture Education). He wanted to establish an international visual language which can be understood cross-cultural and independent on language and class barriers in a transdisciplinary way. Acincı used for the representation of car numbers icons of a VW Bug and for the representation of the cities a mosque (Edirne) and a bridge (Istanbul).

The center of Akınc1's poster is the comparative presentation of two maps which shows the rainfall in the "Rural City Edirne" and the "Urban City Istanbul" in 2017. The overlay of the different rain zones on the maps not too detailed. Therefore, the participants understood mainly quite fast the main information is that the rainfall in the European part of Istanbul was extraordinary high. Above and below the maps are charts in the style of Neurath. The density of the information is quite low. In contrast, Cansaran's poster consist of pure diagrams and tables. The eyecatcher is the redcoloured verbal text "Turkey has no need to build new energy plants, forecasts are mostly wrong!" in the middle of the poster. In other words, he already gave instructions how to read the tables and diagrams. This had significant consequences for the reception of the posters. As described above, all graphic posters were accompanied with texts which gave more detailed information about the topic. In the case of Akınc1's poster, nearly all participants started the reception with the map of the graphic poster. Most of them did not read the text poster beside the graphic poster. The relevance for the life world of the participants arose from the information about Istanbul. Nearly all Turkish participants have already been in Istanbul. They know the problematic situation regarding the car traffic, in particular in the center of Istanbul near the Bosphorus, where car traffic is seen all day, almost every day. The participants from other countries than Turkey know Istanbul less. Therefore, the perceived information needs of these participants were much more related to information about Istanbul than Edirne. Typically, the participants decoded provided information quite fast and started exchanging own experiences with Istanbul or other big cities ("have you ever traveling with public transportation in Istanbul? [...] it is awful [...] I don't have space to move inside the metros." (3B from Pakistan); "I always compare a population of Istanbul with the population of Poland, and in Istanbul are 18 [million citizens], and it is like half of Poland" (3A from Poland)). The most of the recipients did not read the text poster, and started discussing own life experiences and media news. Many of them discussed the activities of Greta Thunberg and the Friday for Future activities combined with criticisms of governmental activities and ignorance. In contrast, the participants dealt with Cansaran's poster in a completely different way. Some of them rejected the reception because of "too many numbers" (22A/B) or dealt in a very sketchy way. Many of them were attracted by the headline but before they started decoding of the tables and graphs, they read the text poster. It revealed that the pure information of charts constitutes a threshold which is difficult to get over. As a consequence, many recipients escape from the topics or fall back on text media which they regard as easier to understand. The catchy text "Turkey has no need to build new energy plants, forecasts are mostly wrong!" helps the access to the topic of the poster but at the same time it avoids the deeper decoding activities, since the main idea is already given. The described results are only a little segment of a broader context. But it reveals that environmental communication cannot provide physical reality as objective facts. It needs to connect phenomena with experiences of the lifeworld. 


\section{Discussion}

The current study examined if PLM is an effective method for youth to learn and process information and knowledge about renewable energy technologies. Consequently, planning, management and implementation of the training concept are based on the interrelation of basic elements and guidelines with a focus on high quality.

The dialogues that were recorded between the students revealed significant information about the peer learning effect in the usage of sustainable energy. As the results showed, PLM is an effective learning methodology since youth access and learn the information with the help of the explanation of the peer and because of explaining the information step-by-step to the peer. In addition, participants used stories and experiences from their own life world to explain and elaborate on the information to their peers to demonstrate the strategy of understanding through connecting the topic with. Next, peers also interacted through a common discovery of the information, thereby showing higher self-determination to acquire the presented information since the role of the teacher is dissolved.

Based on these results we think that the dialogues and quotes indicate and confirm on the one hand the importance of storytelling when considering the public understanding of science. As the current study shows, stories have the opportunity to bridge scientific knowledge with the life world in a catalytic way and can be an effective strategy to decrease the distance between knowledge from the science world and the experiences of the life world [15]. The peer learning strategy in the context of selfdetermination appeared to be more significant since the role of the teacher is dissolved, and more responsibility and determination is activated among students themselves. The learning strategy is negotiated between the partners in an emancipated way, and less influenced by handling a concrete problem or situation that is provided by the teacher, making the learning process intrinsically more determined.

Students learn from others (peers) by actively engaging in large trainee groups to experience practical skills, in combination with appropriate and real time feedback in order to improve their deep understanding and learning of real world aspects. In this case, the important subject of sustainable energy usage [4, 9, 10]. PLM is an effective methodology to reach a higher number of children and young people, with their own communication skills and tools, and, therefore, to create a higher level of awareness on renewable energy. In addition, they also stimulate acquiring practical skills, obtaining professional skills, accepting environmental values, improving language skills, and learn social and cultural competences.

Currently, scientific knowledge is mostly outside of the stock of knowledge of the general population, whereby media can, and should, contribute to the popularization of science, which is a process of decreasing the distance between science world and life world in order to share knowledge and influence the mass to use more sustainable energy [17]. Science as a system has no access to the communicative everyday life practice without medial support, so the main function of media is to enable catalytically the compatibility between the scientific object and existing mental images. Global information need is becoming higher than the individual information need, because of the big distance between lifeworld and science world. Accordingly, beside the interests, 
there has to exist an information need to deal with a scientific topic, which means that, a medium is only regarded as relevant with respect of the acquisition of knowledge, if a recipient has an information need which has to be released through awakening interest, dependent on the distance to the everyday life. Peers increase the interest and need to acquire certain knowledge as the results showed, so PLM can be a very effective methodology to increase the theoretical and practical knowledge about sustainable energy among youth.

The current study has several strengths. First, most participating students considered it as joyful to participate and reported to have learned much about sustainable energy. This was found also among Dutch and international students studying in Tilburg. Second, testing both Turkish and Dutch students gave us the possibility to compare cultural differences between participants from these countries. Because the posters were created taking into account sustainable energy options in Turkey, it was interesting to see how Dutch students perceived the posters, because it is less directly relevant for them. Third, considering that the study was conducted in a real-life exhibition with multiple technological devices to collect the data, the ecological validity of the study is guaranteed.

In contrast, some limitations also exist. First, only a small group of participants were included in the current study. Conducting a study like this is time and energy consuming, therefore it was difficult to obtain more participants. Second, no control groups were established in the current study, so comparing the PLM to other forms of educational methods is difficult. Because the study was exploratory and we interpreted the results qualitatively, we recommend future studies to experimentally test a larger number of participants with a control group to see potential differences in educational settings.

\section{General Conclusion}

This study showed that PLM is an effective and joyful education methodology to inform and activate youth to learn more about sustainable energy. The translation from scientific knowledge to the mass is highly needed in order to modify human's energy behaviour and make it more sustainable. Considering the rapid economic and societal developments in developing countries, and its increased energy usage that develops in parallel, we need to rethink our energy usage immensely and educate youth effectively to learn more about sustainable energies and their usage (see also [5, 6]).

Acknowledgment. This research paper was generated within the scope of the project "POWER: Empowerment of Youth on Renewable Energy for Sustainable Societies", which is funded by the Erasmus+ Program of the European Union 2017-3-TR01-KA205-048402.

\section{References}

1. Chu, S., Majumdar, A.: Opportunities and challenges for a sustainable energy future. Nature 488(7411), 294 (2012) 
2. Beckage, B., Gross, L.J., Lacasse, K., Carr, E., Metcalf, S.S., Winter, J.M., Howe, P.D., Fefferman, N., Franck, T., Zia, A., Kinzig, A.: Linking models of human behaviour and climate alters projected climate change. Nat. Clim. Chang. 8(1), 79 (2018)

3. Urry, J.: Climate change and society. In: Why the Social Sciences Matter, pp. 45-59. Palgrave Macmillan, London (2015)

4. Ito, K.: CO2 emissions, renewable and non-renewable energy consumption, and economic growth: evidence from panel data for developing countries. Int. Econ. 151, 1-6 (2017)

5. United Nations Framework Convention on Climate Change (UNFCCC). https://unfecc.int/ process/the-paris-agreement/status-of-ratification. Accessed 18 Apr 2019

6. IPBES: Nature's dangerous decline 'unprecedented' species extinction rates 'accelerating' (2019). http://campaign.r20.constantcontact.com/render? $\mathrm{m}=1102243211802 \& \mathrm{ca}=2853 \mathrm{ad} 8 \mathrm{~d}$ f207-4e95-adda-5fcc9e7bfc88. Accessed 9 May 2019

7. Kushwaha, G.S., Sharma, N.K.: Green initiatives: a step towards sustainable development and firm's performance in the automobile industry. J. Clean. Prod. 121, 116-129 (2016)

8. Larcher, D., Tarascon, J.M.: Towards greener and more sustainable batteries for electrical energy storage. Nat. Chem. 7(1), 19 (2015)

9. Inglesi-Lotz, R.: The impact of renewable energy consumption to economic growth: a panel data application. Energy Econ. 53, 58-63 (2016)

10. Ozturk, I., Acaravci, A.: CO2 emissions, energy consumption and economic growth in Turkey. Renew. Sustain. Energy Rev. 14(9), 3220-3225 (2010)

11. Barnlund, D.C.: A transactional model of communication. In: Communication Theory, pp. 47-57. Routledge (2017)

12. Moldovan, L.: Innovative method of peer assisted learning by technology and assessment of practical skills. Procedia Technol. 12, 667-674 (2014)

13. Ryan, R.M., Deci, E.L.: Self-determination theory and the facilitation of intrinsic motivation, social development, and well-being. Am. Psychol. 55(1), 68 (2000)

14. Bandura, A., Walters, R.H.: Social Learning Theory, vol. 1. Prentice-hall, Englewood Cliffs (1977)

15. Peschke, L.: Infografiken. Visualität und Wissensaneignung in der mediatisierten Welt. Springer VS, Wiesbaden (2019)

16. Luhmann, N.: Soziale Systeme. Grundriß einer allgemeinen Theorie. Suhrkamp, Frankfurt am Main (1987)

17. Schütz, A., Luckmann, T.: Strukturen der Lebenswelt. UVK, Konstanz (2003). [1979]

18. Habermas, J.: Theorie des kommunikativen Handelns. Band 2: Zur Kritik der funktionalistischen Vernunft. Suhrkamp, Frankfurt am Main (1987)

19. Foskett, D.J.: A note on the concept of relevance. Inf. Storage Retr. 8(2), 77-78 (1972)

20. Lancaster, F.W.: Information Retrieval Systems: Characteristics, Testing and Evaluation. Wiley, New York (1979)

21. Mizzaro, S.: How many relevances in information retrieval? Interact. Comput. 10(3), 305$322(1998)$

22. Hall, S.: The work of representation. In: Hall, S., Evans, J., Nixon, S. (eds.) Representation. Cultural Representations and Signifying Practices, 2nd edn. SAGE, London u.a. (2013)

23. Peschke, L.: Visuality of the Anthropocene. A Concept for a Poster Exhibition. Book on Demand, Norderstedt (2019)

24. Jackson, T.: Renewable Energy: Prospects for Implementation, 1st edn. ButterworthHeinemann (1913). ISBN 978-1-4832-5695-5

25. van Someren, M.W., Barnard, F.Y., Sandberg, J.A.C.: The Think Aloud Method. A Practical Guide to Modelling Cognitive Processes. Academic Press, London (1994)

26. Kelly, G.: The Psychology of Personal Constructs, vol. 1-2. Norton, New York (1955) 
27. Bonarius, H., Holland, R., Rosenberg, S.: Personal Construct Psychology. Recent Advances in Theory and Practice. MacMillan, London (1981)

28. Viney, L.L.: Social science research in the 1990s: the contribution of constructivism. Int. J. Pers. Constr. Psychol. 5(3), 295-305 (1992)

29. Seyfafjehi, S.: Energy resources on mars. In: Peschke, L. (ed.) Visuality of the Anthropocene. A Concept for a Poster Exhibition. Book on Demand, Norderstedt (2019)

30. Yilmaz, A.D.: Serial killer wind turbines? In: Peschke, L. (ed.) Visuality of the Anthropocene. A Concept for a Poster Exhibition. Book on Demand, Norderstedt (2019)

31. Akınc1, N.: Urbanized city through rainfall: how does climate change relate to it? In: Peschke, L. (ed.) Visuality of the Anthropocene. A Concept for a Poster Exhibition. Book on Demand, Norderstedt (2019)

32. Cansaran, O.E.: Power generation of Turkey. An analysis on guesses and facts. In: Peschke, L. (ed.) Visuality of the Anthropocene. A Concept for a Poster Exhibition. Book on Demand, Norderstedt (2019)

33. Neurath, O.: Gesammelte bildpädagogische Schriften. In: Haller, R., Kinross, R. (eds.) Hölder-Pichler-Tempsky, Wien (1991) 